\title{
Time Alignment and Calibration of the LHCb Calorimeter
}

\author{
Miriam Calvo on behalf the LHCb Calorimeter group \\ LIFAELS, Universitat Ramon Llull. Barcelone, Spain
}

\begin{abstract}
The calorimeter system of the LHCb experiment consists of a scintillator layer (SPD), a preshower detector (PS), an electromagnetic calorimeter (ECAL) and a hadronic calorimeter (HCAL), and allows to trigger on hadron, electron and photon candidates. The time synchronization of the 4 sub-detectors studied with cosmic rays, LHC beam injection tests and LHC proton proton collisions is presented. Energy calibration methods for each subsystem are discussed as well.
\end{abstract}

Key words: LHCb, Calorimeter, Calibration, Time alignment, Synchronization

\section{Introduction}

The LHCb experiment $[1,2,3]$ is dedicated to B-physics, and focus on the search of New Physics in CP Violation and rare decays [4]. As $b \bar{b}$ pairs are produced in the same forward or backward direction, the $\mathrm{LHCb}$ detector is a single-arm forward spectrometer.

The calorimeter system [5] plays a key role at the first level of trigger (L0) [6], for which it provides high $p_{T}$ electron, photon and hadron candidates.

In this note we present results on time alignment and preliminary calibration of the calorimeter sub-detectors coming from the commissioning and very first data. For those studies, the ability to readout consecutive crossings for a single trigger (TAE mode) has revealed to be as useful as successful.

For the 2010 LHC run, calibration methods adapted to each sub-detector system are planned.

\section{Calorimeter system}

The LHCb calorimeter is divided into four parts: Scintillator Pad Detector (SPD), Preshower detector (PS), Electromagnetic Calorimeter (ECAL) and Hadronic Calorimeter (HCAL). The lateral dimensions of the calorimeter system are approximately $8 \times 7 \mathrm{~m}^{2}$ and it starts at $12.3 \mathrm{~m}$ from the interaction point with a total depth of $2.7 \mathrm{~m}$

SPD and PS are both scintillator layers of $1.5 \mathrm{~cm}$ thickness, with a lead layer $\left(2.5 X_{0}\right)$ between them. Each one has 6016 scintillating cells where light is collected by WLS fibers and led to MAPMTs of 64 channels. Each plane is divided into three regions: inner, middle and outer, with 4,6 and $12 \mathrm{~cm}$ scintillator square pads, respectively.

The objective of these two detectors at LO is to determine the charged and electromagnetic nature of the ECAL cluster candidate. For electromagnetic clusters, the SPD tags the particle charge for the e $/ \gamma$ separation by comparing the integrated signal with respect to a threshold. The PS provides the e $/ \pi$ separation, as electromagnetic particles start a shower in the lead plate ahead of the Preshower. The SPD multiplicity is used at L0 to require some activity in the detector or to veto events with high charged particle multiplicity when the LHC works at its nominal luminosity. The PS energy is used offline to determine the electron or photon energy while the SPD allows to identify converted photons after the magnet.

ECAL is Shashlik type. Each channel consists of 66 alternating lead-scintillator layers, which corresponds to $25 X_{0}$. It has a projective geometry with SPD and PS, so the 6016 channels are divided in the same three lateral regions. The granularity is small enough to separate significant fraction of $\gamma$ pairs from neutral pion decays and, at the general level, to minimize pile-up effects. In that case there is one PMT per channel. The energy resolution is around $\sigma / E(\mathrm{GeV})=10 \% / \sqrt{E} \otimes 1 \%$ [7]. ECAL is also important for the offline reconstruction of B-decays containing $\pi^{0}$, photons or electrons.

HCAL is a sampling iron-scintillator calorimeter of $5.6 \lambda_{I}$ thickness with structure arranged along the beam axis. In this case the detector is divided in two regions, inner and outer with 13 and $26 \mathrm{~cm}$ sizes, respectively. In total, 1488 cells readout also with WLS and PMTs. The energy resolution is on the level of $\sigma / E(G e V)=80 \% / \sqrt{E} \otimes 10 \%$ [8]. It has a self-calibration system of ${ }^{137} \mathrm{Cs}$ radioactive source embedded into the calorimeter structure.

The four sub-detectors can monitor the PMT response and stability with LED systems, also used to spot noisy and dead channels.

Because of similarity of input signals and functionalities, common electronic system for the ECAL and HCAL is used, based on a dead-timeless and low pedestal integrator system using delay lines, followed by ADCs and pipeline buffers. Similar electronics are used for SPD and PS, with two alternating subchannels and spill-over correction.

For all calorimeters $>99.9 \%$ channels are working by begin- 
ning of 2009 data taking.

\section{Commissioning and LHC proton proton collisions}

Because of its particular geometry, the use of cosmic rays in $\mathrm{LHCb}$ is a real challenge, limited to the commissioning of the large sub-detectors (Outer Tracker, Calorimeter, Muon system) and trigger. Nonetheless, 4 millions of events (from Summer 2008 to Autumn 2009) were recorded.

The cosmic trigger is based on ECAL and HCAL coincidence (or Muon), with a rate of $10 \mathrm{~Hz}$. As cosmic rays come from the top of the detector, the slope of the track provides the forward or backward direction, and then time-of-flight corrections could be applied to perform time alignment.

Another useful tool for commissioning was the LHC beam injection events. A proton beam was dumped around $340 \mathrm{~m}$ before the detector, also known as Transfer line External beam Dump (TED) events. There were shots of around $5 \times 10^{9}$ protons every 48 seconds. A high flux of particles was then produced with approximately 10 particles $/ \mathrm{cm}^{2}$ in the center of the shower. This shower was a bit displaced from the LHCb beam axis, and coming from the backward part of the detector, so in the reverse direction from proton proton collisions, not ideal for absolute synchronization. The events were triggered by requiring the SPD multiplicity to be higher than 10 .

First LHC proton proton collisions took place in November 23, 2009 at a center of mass energy of $900 \mathrm{GeV}$. After some days with collisions at this energy, also collisions at $\sqrt{s}=2.36$ $\mathrm{TeV}$ were held in December 2009.

The Minimum Bias trigger required a HCAL candidate and the SPD multiplicity to be higher than 2 (or Muon system or backward VELO planes). The amount of data collected is summarized in Table 1. The main use of 2009 data for the Calorimeter system was to perform the absolute time alignment with the LHC beam.

\begin{tabular}{|c|c|c|}
\hline Beam energy & Mode & Number of triggers \\
\hline \hline $450 \mathrm{GeV}$ & $1 \mathrm{BX}$ & $450 \mathrm{k}$ \\
\hline $450 \mathrm{GeV}$ & TAE & $115 \mathrm{k}$ \\
\hline $1.18 \mathrm{TeV}$ & $1 \mathrm{BX}$ & $34.5 \mathrm{k}$ \\
\hline $1.18 \mathrm{TeV}$ & TAE & $22 \mathrm{k}$ \\
\hline
\end{tabular}

Table 1: Number of triggered events of proton proton collisions.

\section{Time alignment}

The detector signals are integrated each $25 \mathrm{~ns}$. The integration window has to be adjusted such as for each subdetector the maximum amount of signal is collected.

The integrated signal depends on the starting time of integration of the $25 \mathrm{~ns}$ window. This time is referred as $d T 0$ or delay hereafter. Left Fig. 1 shows the dependence of ECAL signal with the delay.

The delays can vary among different parts of a detector due to differences of fiber and cable lengths, PMT HV and particle

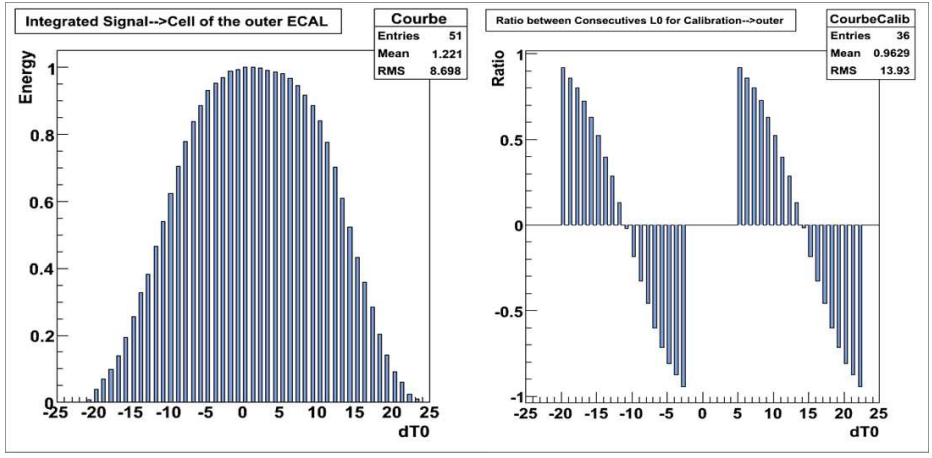

Figure 1: ECAL integrated signal as a function of the time of integration (left) and energy asymmetry (right).

time of flight from the interaction point. Comparing the signal deposited in two consecutive bunch crossings, the delay can be estimated. This is done by defining an asymmetry:

$$
\operatorname{Asym}(T 0 / \operatorname{Prev} 1)=\frac{E_{T 0}-E_{\text {Prev } 1}}{E_{T 0}+E_{\text {Prev } 1}}
$$

where $E_{T 0}$ is the energy in the current bunch crossing and $E_{\text {Prev1 }}$ the energy in the previous one. Also the asymmetry with respect to the next bunch crossing can be used. Right Fig. 1 shows the asymmetries for ECAL. Note that the asymmetry cancels around $12.5 \mathrm{~ns}$ and it is approximately linear in this region. For HCAL these curves are very similar.

The method to synchronize is to shift the integration window to the linear region. Then, from a single measurement the timings of the cells are derived.

For SPD and PS, the synchronization is performed by group of 64 channels (one PMT electronic card), not by cell. The method in this case is also based in the use of asymmetries, which are different from ECAL due to different pulse shapes. For the SPD, as it is a binary detector, the study is done in terms of occupancies and not deposited energy.

\subsection{Cosmic rays}

Cosmic rays arrive at any time within the integration window, so they were not useful to synchronize ECAL and HCAL themselves. However, the track of the muon was reconstructed with ECAL and HCAL information and extrapolated to the SPD/PS planes. The time when the particle crossed within the $25 \mathrm{~ns}$ was provided by the asymmetries measurement of Ecal or Hcal shared energy in consecutive bunch crossings. A relative synchronization of SPD and PS around \pm 2 ns was achieved. Fig 2 shows the corrections to the delays of each SPD card (VFE) obtained from $1 \mathrm{M}$ cosmic events.

\subsection{TED events}

In the TED events particles arrive at the same time. Computing asymmetries around the linear region $(-12 \mathrm{~ns})$, an intradetector time alignment was performed for ECAL, HCAL and PS (now independently from ECAL and HCAL). Only relative synchronization within each detector was done because of the reversed direction. As not all calorimeter cells were fired by the 


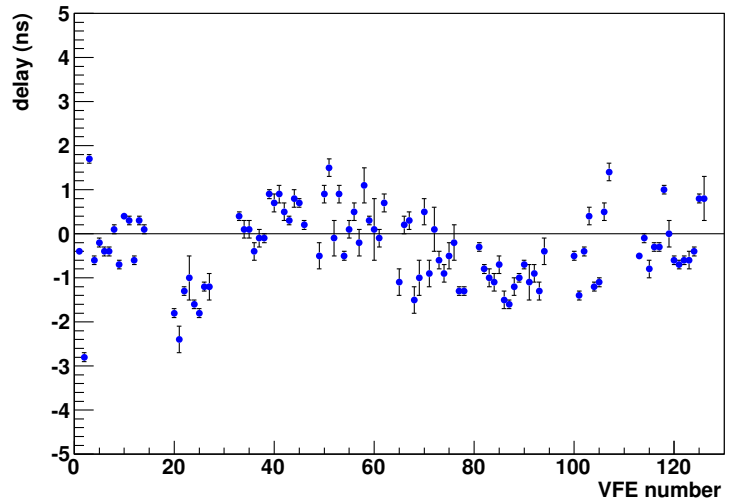

Figure 2: Correction to the delays measured from cosmic rays for each SPD VFE in September 2009. Fit errors are shown.

particles, $60 \%$ of cells were finely synchronized in June and October 2009. For SPD, during the TED events in October 2009, a delay scan was performed with half detector, when the other half was used as trigger. From the asymmetries obtained, cosmic results were confirmed.

\subsection{Proton proton collisions}

Finally, absolute synchronization with LHC beam was performed with proton proton collisions in December 2009. With the available amount of TAE data, 92\% ECAL cells and $87 \%$ HCAL cells were synchronized with a precision below the ns. With 2010 data it is expected to check the final time alignment and signal stability within small time misalignment.

For both SPD and PS a delay scan was performed, with 11 steps ( $\approx 4 \mathrm{k}$ events/step). Fig. 3 shows the PS signal of a Front End card as a function of the delay and the corresponding asymmetry. The expected synchronization achieved in both detectors is $\pm 1 \mathrm{~ns}$.
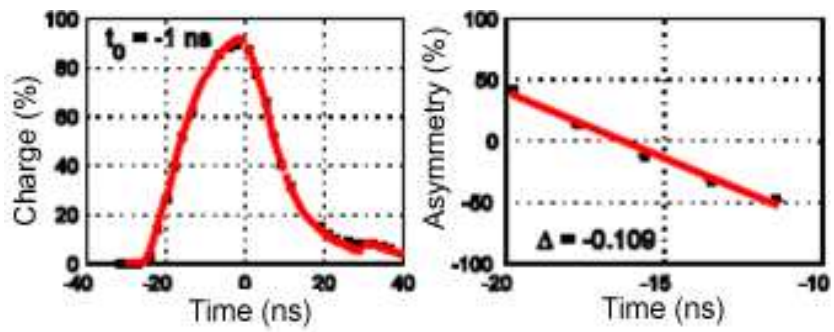

Figure 3: Signal vs delay for a PS FEB from proton proton collisions (left) and Next1/T0 asymmetry (right).

\section{Energy calibration}

From the known PMT gain behavior, the inital energy intercalibration is around $10 \%$ for all sub-detectors. The number of photoelectrons per unit of energy is known from test beams. Several steps for finer calibration are foreseen. First, equalize the cell energy response and then, perform the absolute energy calibration by means of different methods for each sub-detector.
Once calibrated, the PMT response can be followed by the LED system.

\section{1. $S P D$}

The SPD is a binary detector, so it has not straight MIP calibration. The aim is to provide a resolution in the MIP position smaller than $5 \%$, limited by the electronics. The method is to use tracks to compute cell efficiency at a given threshold and compare with theoretical value, shown in Fig. 4. It has to be done per cell, so around $2 \mathrm{M}$ collision data with thresholds at 0.8 and 1 MIP (sensitive region) are required. From the limited samples of tracks from cosmics and 2009 proton proton collisions a dispersion around $10 \%$ was measured among VFEs.

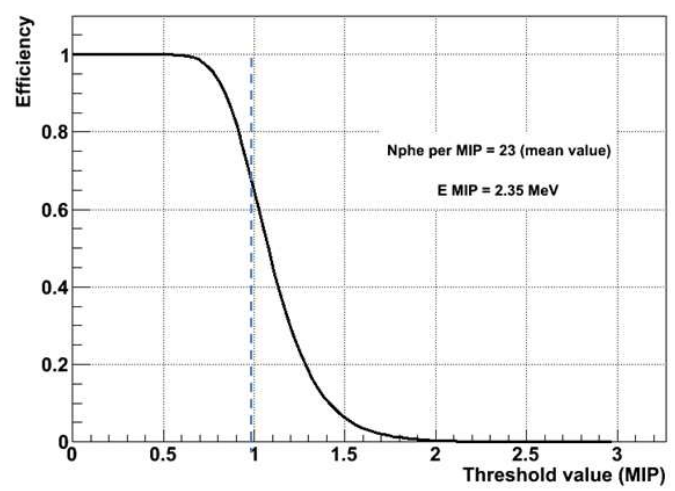

Figure 4: Track efficiency vs SPD threshold. Theoretical curve.

\section{2. $P S$}

With the 4 M cosmics, the MIP energy distribution was fitted per channel, taking into account the correction for different incident angles of the cosmic muons. These data were used to uniformize the channels response, measuring the corrective factors to get the same MIP value per ADC. PMT HVs were also equalized to achieve a $10 \%$ intercalibration.

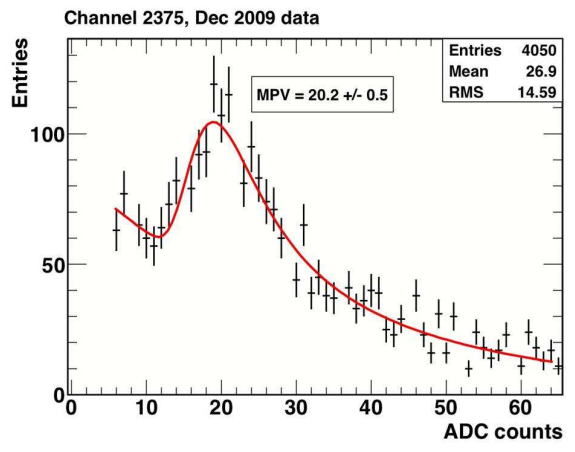

Figure 5: Fit of the MIP energy deposit in a PS cell. 2009 collision data.

With proton proton collisions the MIP signal is fitted by channel as shown in Fig. 5. With 2009 data, 20\% differences between inner and outer peaks were measured. 


\section{3. $E C A L$}

The intercalibration was estimated to be around $8 \%$ at start. The first step for finer calibration is to use the energy flow method [9] that allows a relative intercalibration of $5 \%$ with $4 \mathrm{M}$ events. This cell equalization consists on computing the average transverse energy of a given cell and compare to its neighbours assuming a smooth energy flow, and obtain corrective factors. The method can be used also for PS and HCAL.

The absolute energy calibration, up to $1 \%$ can be achieved using $\pi^{0}$. For electron and photon energy estimation, cluster energy corrections are needed:

$$
E_{\text {rec }}=\alpha E_{\text {cluster }}+\beta E_{P S}
$$

where $\alpha$ depends on barycenter position inside the cluster and inside the module and $\beta$ is the factor applied to the Preshower energy.

Two approaches are under study to use $\pi^{0}$ for calibration. A first one is an iterative procedure based on $\pi^{0}$ mass constraint issued from two separate photons and the second method consists of a global minimization of the distance of each candidate to the PDG mass. Both aim to find each cell calibration coefficient with a precision close to $1 \%$. For 2009 data one can already give rough calibration constants for each area of the calorimeter, used to reconstruct $\pi^{0}$ and $\eta$ as shown in Fig. 6. For 2010 data, $200 \mathrm{M}$ events are needed to calibrate all cells at $1 \%$.

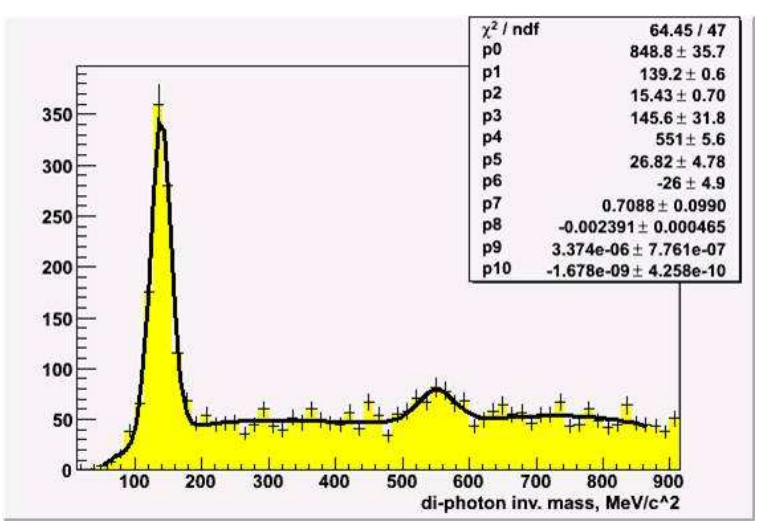

Figure 6: $\pi^{0}$ and $\eta$ reconstructed from proton proton collisions.

The PS $\beta$ correction factor is estimated minimizing the $\pi^{0}$ mass width, distinguishing between converted or non-converted photons from the $\pi^{0}$ decay.

A cross-check of the calibration can be done studying the energy-to-momentum ratio $(\mathrm{E} / \mathrm{p})$ of electrons. It requires precise information from the tracking system and a pure electron sample, obtained from $\mathrm{J} / \psi$ or photon conversions.

\section{4. $H C A L$}

With the radioactive source, an intercalibration of 3-4\% was confirmed. Calibration runs are done regularly, every one or two months. The calibration is followed in between with the LED response.

With proton proton collisions, the absolute energy scale can be verified. Fig. 7 shows the E/p ratio from the HCAL response to a charged particle. There is a nice agreement between MC and data.

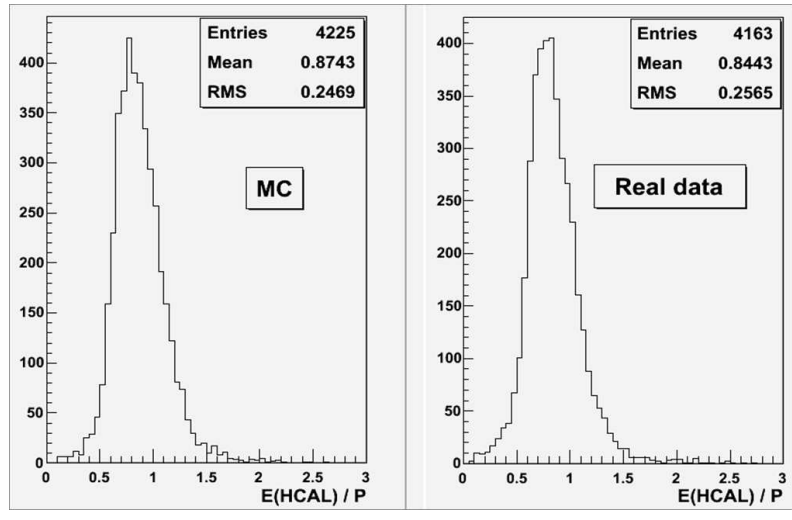

Figure 7: E/p ratio of charged particles at HCAL from MC (left) and 2009 collisions (right).

\section{Conclusions}

The calorimeter system provided a smooth trigger for 2009 data. A previous relative time alignment was performed with cosmic rays and TED events, but the absolute synchronization was achieved with first collisions.

Current energy intercalibration is around 10\% for SPD and PS, $8 \%$ for ECAL and $4 \%$ for HCAL.

In order to perform absolute energy calibration, different methods are planned for each sub-detector. 5\% resolution is expected for SPD measuring efficiencies on tracks and for PS, using MIPs. Several steps are envisaged for ECAL: energy flow, calibration with $\pi^{0}$ and electrons to achieve $1 \%$ calibration. For HCAL, the use of tracks to validate the current calibration is foreseen.

\section{Acknowledgments}

This work is supported by the Spanish MICINN under contract FPA2008-06271-c02-02 and Juan de la Cierva program. Especial thanks to Marie-Noelle Minard for her suggestions and fruitful comments.

\section{References}

[1] LHCb Collaboration. LHCb Technical Proposal. CERN-LHCC-98-004

[2] LHCb Collaboration. LHCb Reoptimized Detector. Design and Performance. CERN-LHCC-2003-030

[3] LHCb Collaboration. The LHCb detector at the LHC. Journal of Instrumentation. 2008 JINST 3 S08005.

[4] LHCb Collaboration. Roadmap for selected key measurements of LHCb. LHCb-PUB-2009-029.

[5] LHCb Collaboration. LHCb Calorimeters Technical Design Report. CERN-LHCC-2000-036

[6] LHCb Collaboration. LHCb Trigger Technical Design Report. CERNLHCC-2003-031

[7] A. Arefiev et al. Beam test results of the LHCb electromagnetic calorimeter. $\mathrm{LHCb}-2007-149$

[8] C. Coca et al. The Hadron Calorimeter Prototype Beam Test Result. LHCb-2000-036

[9] I. Belyaev and K. Voronchev. Energy flow calibration of LHCb ECAL. LHCb-2006-051 